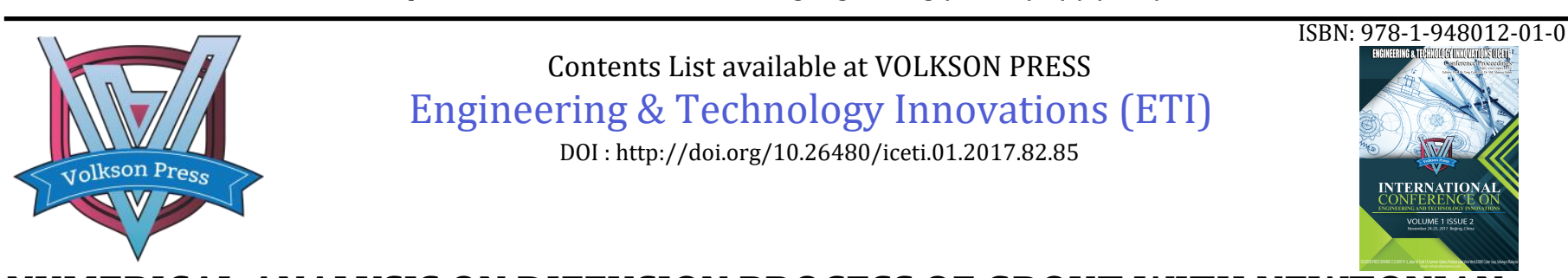

\title{
NUMERICAL ANALYSIS ON DIFFUSION PROCESS OF GROUT WITH NEWTONIAN BEHAVIOUR IN RECTANGULAR PLANE FISSURE
}

\author{
Xiaoqian Luo, Xiaolong Li* \\ School of Water Conservancy and Environment, Zhengzhou University, \\ No.100 of Science Road, Zhengzhou, China \\ *zzulx11977@163.com
}

This is an open access article distributed under the Creative Commons Attribution License, which permits unrestricted use, distribution, and reproduction in any medium, provided the original work is properly cited

\section{ARTICLE DETAILS}

\section{Article History:}

Received 02 october 2017 Accepted 06 october 2017 Available online 11 october 2017

Keywords: grouting, fissure, finite volume method, VOF, numerical simulation

\section{ABSTRACT}

Grouting is widely used to reduce water flow through rock masses and improve in situ rock quality. In recent decades, there has always been a significant requirement to understand the mechanism behind grout spread in jointed rock. In this study, the flow process of a Newtonian grout in a rectangular plane fissure initially filled with air is numerically investigated by using the finite volume method (FVM) and the volume of fluid (VOF) technique. The results show that before the flow front reaches the side wall, the grout movement exhibits a radial flow, and after that, the grout flow direction changes and it flows along the y-direction, parallel to the side wall, with an uneven flow front. With the gradual increase of grout propagation length, the flow front tends to be flat and finally becomes a horizontal straight line. The pressure gradient of the flow field continuously decreases with the enlargement of grout spread area, as results in a rapid decline of grout rate.

\section{INTRODUCTION}

Grouting is widely used to seal tunnels and improve rock conditions under dams and foundations and, in this regard, various grouting materials have been developed [1,2]. Several grouts with Newtonian behaviour, such as silicates and acrylates, have been used for a long time. One of the most important challenges in penetration grouting is computation of the grout's penetration depth around borehole in the design or analysis. Numerical modelling of grout propagation in fracture geometries is one means of achieving this goal. Some researchers have analyzed the grout spread with numerical methods. Hassler et al. presented a model for simulating grout propagation in jointed rock use the single channel as the basic component [3]. Eriksson et al. developed a numerical method to estimate grout spread area in the rock joints considering grout filtration and varying fracture aperture [4]. Yang et al. have carried out a numerical simulation using a stochastic joint network and indicated the effects of the joint properties on grout penetration depth [5]. Saeidi et al. proposed a numerical model to predict grout flow and penetration length into the jointed rock mass using UDEC and considering joint roughness and strength [6].

In the numerical models mentioned above, the single channel is the basic component of the joint network generated stochastically or regularly, and this means that the grout propagation is simplified as a one-dimensional flow. However, in practice two-dimensional flow in rock joints would occur in a three-dimensional structure of the rock mass. Several researchers, as Funehag and Gustafson [7] and EI-Tani [8] have proposed analytical solutions by ideally regarding grout movement as a radial flow between non-deforming infinite parallel plates. However, the actual single fracture in rock mass has a limited size and its shape usually can be seen as a rectangle but not a disc, hence the analytical methods possess a limited applicability when using them for grouting design or analysis.

In this study, a numerical model was proposed to predict grout flow into the jointed rock mass. The numerical model use finite volume method (FVM) for discretizing the governing equations of flow and volume of fluid (VOF) technique for tracking the interface between grout and air. Under a constant grout pressure, the flow process of a grout with Newtonian behaviour in a rectangular plane fissure is investigated using the presented numerical model. The grout diffusion process and pressure distribution of flow field is analyzed and the variation of grout flow rate with time is also discussed.

\section{Mathematical modelling}

\subsection{Conservation of mass}

The grout is treated as an incompressible Newtonian fluid with constant density, and the continuity equation is given as:

$$
\operatorname{div}(\rho \mathbf{u})=0
$$

Here $\mathbf{u}$ is the velocity vector, and $\rho$ is the density.

\subsection{Conservation of momentum}

The equation governing the motion of the system is given by

$$
\begin{aligned}
& \frac{\partial(\rho u)}{\partial t}+\operatorname{div}(\rho u \mathbf{u})=-\frac{\partial p}{\partial x}+\operatorname{div}(\mu \operatorname{grad} u)+S_{M x} \\
& \frac{\partial(\rho v)}{\partial t}+\operatorname{div}(\rho v \mathbf{u})=-\frac{\partial p}{\partial y}+\operatorname{div}(\mu \operatorname{grad} v)+S_{M y} \\
& \frac{\partial(\rho w)}{\partial t}+\operatorname{div}(\rho z \mathbf{u})=-\frac{\partial p}{\partial z}+\operatorname{div}(\mu \operatorname{grad} w)+S_{M z}
\end{aligned}
$$

Here $u, v$ and $w$ are the $x$-component, $y$-component, and $z$-component of velocity vector u respectively, $t$ is time, $S_{M}$ is the momentum source, $p$ is pressure, and $\mu$ is the shear viscosity.

\subsection{VOF function}

In order to track the interface between grout and air, the fractional volume function is defined such that 
$f(x, t)=\left\{\begin{array}{cc}1 & \text { for the point }(\mathbf{x}, t) \text { filled with grout } \\ 0 & \text { for the point }(\mathbf{x}, t) \text { filled with air }\end{array}\right.$

This function is governed by the following scalar advection equation.

$\frac{\partial f}{\partial t}+\mathbf{u} \cdot \operatorname{div}(f)=0$

The interface is located within the cells whose average value of $f$ lies between 0 and 1 . For these cells, material properties, viscosity and density, are interpolated linearly using the value of $f$. For partially filled cells, material properties such as viscosity and density are interpolated using

$$
\begin{aligned}
& \mu=\mu_{\text {grout }} f+\mu_{\text {air }}(1-f) \\
& \rho=\mu_{\text {grout }} f+\rho_{\text {air }}(1-f)
\end{aligned}
$$

\section{Numerical formulation}

In this study, a pressure-based FVM scheme for structured meshes and the SIMPLE algorithm are used [9]. All variables are stored at the cell centres, and the momentum interpolation method is used to prevent checkerboard pressure mode.

\subsection{Pressure correction equation}

Integrating the continuity equation (1) over the control volume and applying the divergence theorem, we obtain

$$
\begin{aligned}
& {\left[(\rho u A)_{e}-(\rho u A)_{w}\right]+\left[(\rho v A)_{n}-(\rho v A)_{s}\right]} \\
& +\left[(\rho v A)_{t}-(\rho v A)_{b}\right]=0
\end{aligned}
$$

Here $A$ is the cross-sectional area of the control volume face, the notation $w, e, s, n, b$ and $t$ refer to the west, east, south, north, bottom and top cell face respectively.

By using a fully implicit time discretization, we obtain the discretized continuity equation as

$$
\begin{gathered}
a_{I, J, K} p_{I, J, K}^{\prime}=a_{I+1, J, K} p_{I+1, J, K}^{\prime}+a_{I-1, J, K} p_{I-1, J, K}^{\prime} \\
+a_{I, J+1, K} p_{I, J+1, K}^{\prime}+a_{I, J-1, K} p_{I, J-1, K}^{\prime} \\
+a_{I, J, K-1} p_{I, J, K-1}^{\prime}+a_{I, J, K+1} p_{I, J, K+1}^{\prime}+b_{I, J, K}^{\prime} \\
\text { Where } \begin{array}{r}
a_{I, J, K}= \\
\quad a_{I+1, J, K}+a_{I-1, J, K}+a_{I, J+1, K} \\
+a_{I, J-1, K}+a_{I, J, K-1}+a_{I, J, K+1}
\end{array} \\
\text { and } \begin{aligned}
b_{I, J, K}^{\prime}=(\rho u A)_{i, J, K}-(\rho u A)_{i+1, J, K}+(\rho v A)_{I, j, K} \\
-(\rho v A)_{I, j+1, K}+(\rho v A)_{I, J, k-1}-(\rho v A)_{I, J, k+1}
\end{aligned}
\end{gathered}
$$

With neighbour coefficients:

$$
\begin{aligned}
& a_{I+1, J, K}=(\rho d A)_{i+1, J, K}=(\rho d A)_{e}, \\
& a_{I-1, J, K}=(\rho d A)_{i-1, J, K}=(\rho d A)_{w}, \\
& a_{I, J+1, K}=(\rho d A)_{I, j+1, K}=(\rho d A)_{n}, \\
& a_{I, J-1, K}=(\rho d A)_{I, j-1, K}=(\rho d A)_{s}, \\
& a_{I, J, K+1}=(\rho d A)_{I, J, k+1}=(\rho d A)_{t}, \\
& a_{I, J, K-1}=(\rho d A)_{I, J, k-1}=(\rho d A)_{b} .
\end{aligned}
$$

Where $I, J$, and $K$ are the numbers of the control volume in the $x$-, $y$ - and $z$ directions respectively and $i, j$, and $k$ are the numbers of the control volume face in the $x-, y$ - and $z$-directions respectively.

\subsection{Discretized momentum equation}

By integrating the momentum equation (2) over a control volume and a finite time step $\Delta t$, and replacing the volume integrals of the convective and diffusive terms with surface integrals we obtain

$$
\begin{aligned}
& \int_{t}^{t+\Delta t} \int_{\Delta v} \frac{\partial(\rho \phi)}{\partial t} \mathrm{~d} V \mathrm{~d} t+\int_{t}^{t+\Delta t} \int_{\Delta v} \operatorname{div}(\rho \boldsymbol{u} \phi) \mathrm{d} V \mathrm{~d} t= \\
& \int_{t}^{t+\Delta t} \int_{\Delta v} \operatorname{div}(\mu \operatorname{grad} \phi) \mathrm{d} V \mathrm{~d} t+\int_{t}^{t+\Delta t} \int_{\Delta v} S \mathrm{~d} V \mathrm{~d} t
\end{aligned}
$$

Where $\phi$ is a general variable, and by setting $\phi$ equal to $u, v$ and $w$, we obtain special forms of three momentum equations.

The integral of transient term can be written as

$$
\int_{t}^{t+\Delta t} \int_{\Delta v} \frac{\partial(\rho \phi)}{\partial t} \mathrm{~d} V \mathrm{~d} t=\rho_{P}^{0}\left(\phi_{P}-\phi_{P}^{0}\right) \Delta V
$$

Here $\Delta V$ is the volume of the control volume, and the superscript ' 0 ' refers to the variable value at time $t$. By applying the divergence theorem and replacing the volume integrals with surface integrals, the convective terms can be written as

$$
\begin{gathered}
\int_{t}^{t+\Delta t} \int_{\Delta v} \operatorname{div}(\rho \boldsymbol{u} \phi) \mathrm{d} V \mathrm{~d} t=\int_{t}^{t+\Delta t}\left[(\rho u A)_{e} \phi_{e}-(\rho u A)_{w} \phi_{w}\right. \\
\left.+(\rho v A)_{n} \phi_{n}-(\rho v A)_{s} \phi_{s}+(\rho v A)_{t} \phi_{t}-(\rho v A)_{b} \phi_{b}\right] \mathrm{d} t
\end{gathered}
$$

Similarly, by replacing the volume integrals of the diffusive terms with surface integrals we obtain

$$
\begin{aligned}
& \int_{t}^{t+\Delta t} \int_{\Delta v} \operatorname{div}(\mu \operatorname{grad} \phi) \mathrm{d} V \mathrm{~d} t=\int_{t}^{t+\Delta t}\left[\left(\mu \frac{\partial \phi}{\partial x} A\right)_{e}-\left(\mu \frac{\partial \phi}{\partial x} A\right)_{w}\right. \\
& \left.+\left(\mu \frac{\partial \phi}{\partial x} A\right)_{n}-\left(\mu \frac{\partial \phi}{\partial x} A\right)_{s}+\left(\mu \frac{\partial \phi}{\partial x} A\right)_{t}-\left(\mu \frac{\partial \phi}{\partial x} A\right)_{b}\right] \mathrm{d} t \text { (12) }
\end{aligned}
$$

Using a fully implicit time derivative and discretizing the convective and diffusive term, we obtain a discrete momentum equation as follows:

$a_{P} \phi_{P}=a_{E} \phi_{E}+a_{W} \phi_{W}+a_{N} \phi_{N}+a_{S} \phi_{S}+a_{B} \phi_{B}+a_{T} \phi_{T}+b$

Where $a_{P}=a_{W}+a_{E}+a_{S}+a_{N}+\left(F_{e}-F_{w}\right)+\left(F_{n}-F_{s}\right)+a_{P}^{0}-S_{P} \Delta V$ $a_{P}^{0}=\rho_{P}^{0} \Delta V / \Delta t$, and $W, E, S, N, B$ and $T$ denote the control volume $P^{\prime} \mathrm{s}$ six neighbouring control volumes, the west, east, south, north, bottom and top respectively.

The neighbour coefficients of this equation for the power-law differencing scheme are as follows:

$$
\begin{aligned}
& a_{W}=D_{w} \max \left[0,\left(1-0.1\left|F_{w} / D_{w}\right|\right)^{5}\right]+\max \left[0, F_{w}\right] \\
& a_{E}=D_{e} \max \left[0,\left(1-0.1\left|F_{e} / D_{e}\right|\right)^{5}\right]+\max \left[0,-F_{e}\right] \\
& a_{S}=D_{s} \max \left[0,\left(1-0.1\left|F_{s} / D_{s}\right|\right)^{5}\right]+\max \left[0, F_{s}\right] \\
& a_{N}=D_{n} \max \left[0,\left(1-0.1\left|F_{n} / D_{n}\right|\right)^{5}\right]+\max \left[0,-F_{n}\right] \\
& a_{B}=D_{b} \max \left[0,\left(1-0.1\left|F_{b} / D_{b}\right|\right)^{5}\right]+\max \left[0, F_{b}\right] \\
& a_{T}=D_{t} \max \left[0,\left(1-0.1\left|F_{t} / D_{t}\right|\right)^{5}\right]+\max \left[0,-F_{t}\right]
\end{aligned}
$$

In the above expressions the values of $F$ and $D$ are calculated with the following formulae:

$$
\begin{aligned}
& F_{w}=(\rho u)_{w} A_{w}, F_{e}=(\rho u)_{e} A_{e}, F_{n}=(\rho v)_{n} A_{n} \\
& F_{s}=(\rho v)_{s} A_{s}, F_{b}=(\rho v)_{b} A_{b}, F_{t}=(\rho v)_{t} A_{t} \\
& D_{w}=\mu_{w} A_{w} /(\delta x)_{W P}, D_{e}=\mu_{e} A_{e} /(\delta x)_{P E}, D_{n}=\mu_{n} A_{n} /(\delta y)_{P N} \\
& D_{s}=\mu_{s} A_{s} /(\delta y)_{S P}, D_{b}=\mu_{b} A_{b} /(\delta z)_{B P}, \\
& D_{t}=\mu_{t} A_{t} /(\delta z)_{P T}
\end{aligned}
$$


Where $\delta x, \delta y$ and $\delta z$ denote the distances between the neighbouring control volume center in the $x$-, $y$ - and $z$-directions respectively, and the notation $W, E, S, N, B$ and $T$ refer to the west, east, south, north, bottom and top neighbouring control volume respectively.

\section{Solving fractional volume function}

After solving for the distribution of velocity, the new position of the interface must be updated by solving the fractional volume equation. In this study, the Youngs' VOF method is applied to solve this problem [10].

\section{Results and discussion}

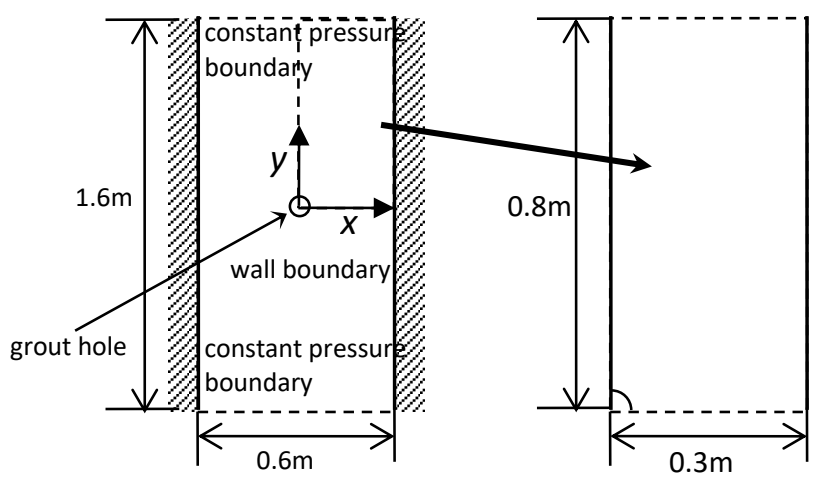

(a)

(b)

Figure 1. Geometry of plane fissure (a) and the simulation domain (b)

The geometry of plane fissure is a three-dimensional rectangular cavity with four walls and two outlets and the dimension is $1.6 \times 0.6 \times 0.0004 \mathrm{~m}^{3}$, as is shown in Figure 1(a). Its left-hand side, right-hand side, front surface and back surface are walls while the top and bottom surface are outlets. A circular grouting hole was set in the center of the plane fissure with a $2 \mathrm{~cm}$ diameter. The fissure is initially filled completely with air. The grout with a density of $1400 \mathrm{Kg} / \mathrm{m}^{3}$ and a viscosity of 0.5 pa.s is injected into the fracture through the hole. The initial pressure and velocity field are set to zero. The grouting pressure is a constant, $10 \mathrm{kPa}$.

To save computational time, only one quarter of the fissure is actually considered in the simulation. As the fissure is symmetric, this is a suitable reduction of the simulation domain. Figure 1(b) shows this simulation domain which is divided into 2400 brick elements.

In order to solve the grout flow process, several assumptions are needed. The grout is modelled as a Newtonian fluid. For solving the empty domain which is initially filled with air, some additional assumptions are needed. The air is assumed to be an incompressible Newtonian fluid. Laminar flow is assumed. The density of air is about $1.25 \mathrm{Kg} / \mathrm{m}^{3}$ and its viscosity is 0.00001 pa.s.

Figure 2 shows the grout spread area at different times. It can be observed that the area filled by grout enlarges gradually over time, the interface between the grout and the air keeps moving with the continuous injection of grout, and finally the flat fracture is fully filled with the grout. Before the flow front reached the left and right wall, the shape of the region that has been filled by grout is approximately a circle and the grout flow exhibits a centrosymmetric radial flow. After that, the grout flow changes and it flows along the y direction, parallel to the side wall, with an uneven flow front. With the continuous enlargement of grout spread area, the flow front tends to be flat and almost becomes a horizontal straight line when the elapsed time is $35 \mathrm{~s}$.

Figure 3(a)-(c) illustrates the pressure distribution of the flow field at time $2.0 \mathrm{~s}, 15 \mathrm{~s}$ and $35 \mathrm{~s}$. It can be found that the maximum pressure value always lies in the grouting hole and is equal to grouting pressure, while the minimum one, zero, lies in the place where the grout has not reached. With a gradual increase of the grout spread area, the space between the neighbouring pressure contours increases correspondingly, as reveals a tendency of continuous decrease in the pressure gradient.

Figure 4 depicts the development of grout rate with grouting time. The grout rate decreased rapidly with respect to time, and this is a normal reflect of continuously decreased pressure gradient and flow velocity.

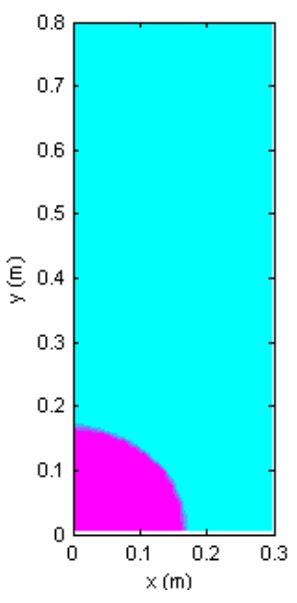

(a) time: $2.0 \mathrm{~s}$

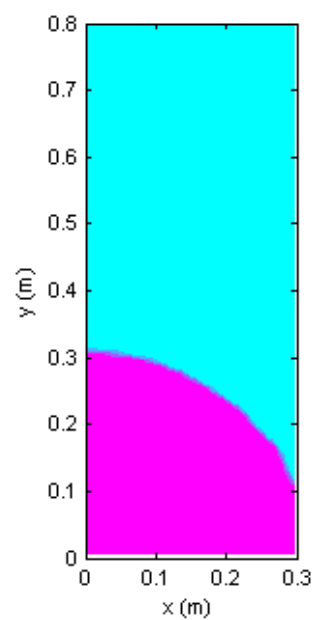

(c) time: $8.5 \mathrm{~s}$

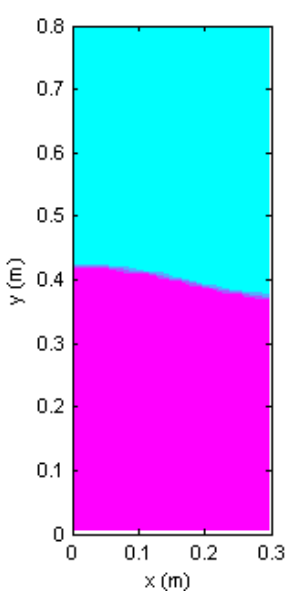

(e) time: $15 \mathrm{~s}$

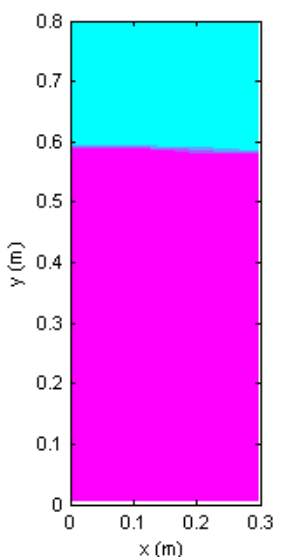

(g) time: $25 \mathrm{~s}$

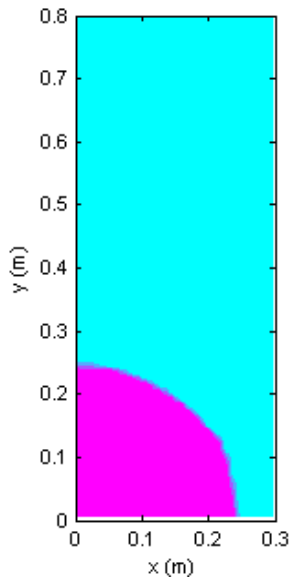

(b) time: $5.0 \mathrm{~s}$

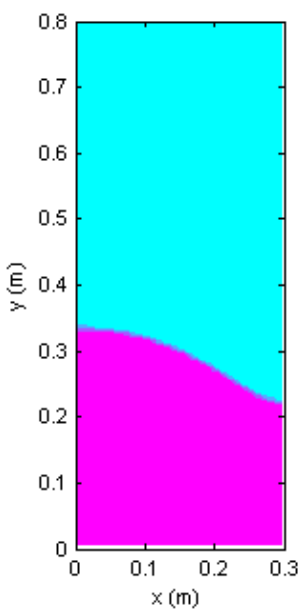

(d) time: $10 \mathrm{~s}$

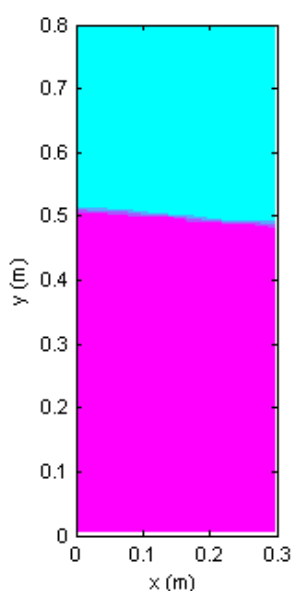

(f) time: $20 \mathrm{~s}$

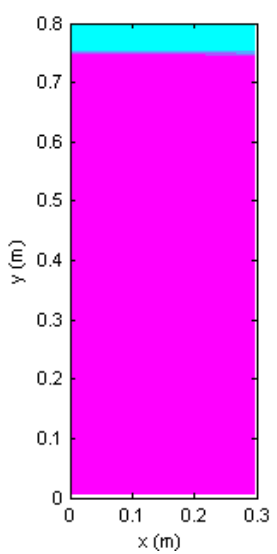

(h) time: $35 \mathrm{~s}$ 
Figure 2. Grout spread area at different times

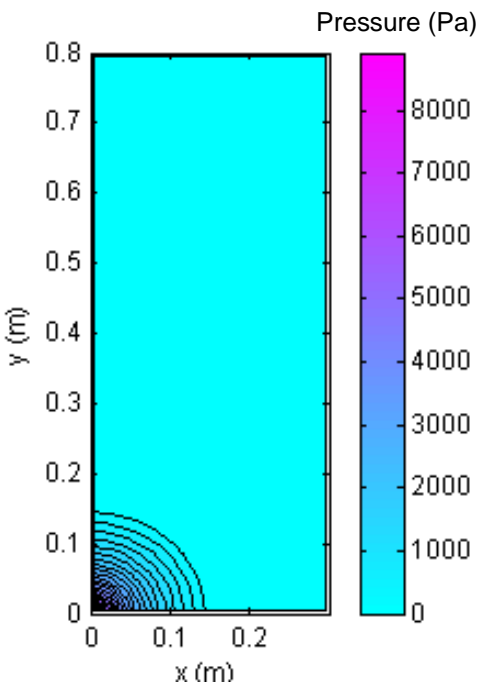

(a) time: $2.0 \mathrm{~s}$

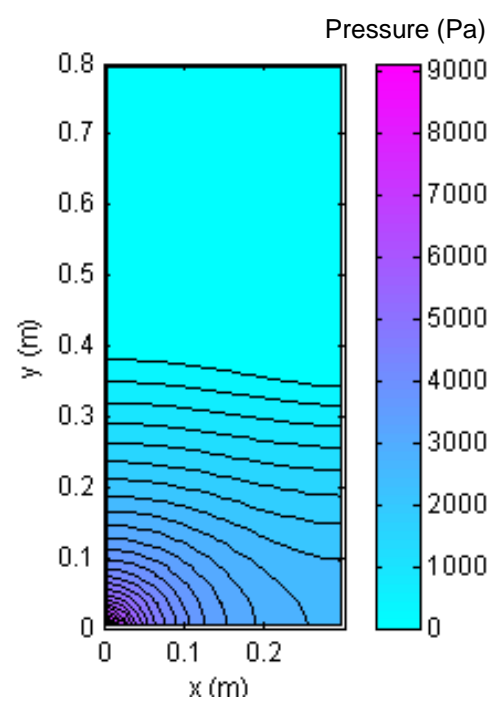

(b) time: $15 \mathrm{~s}$

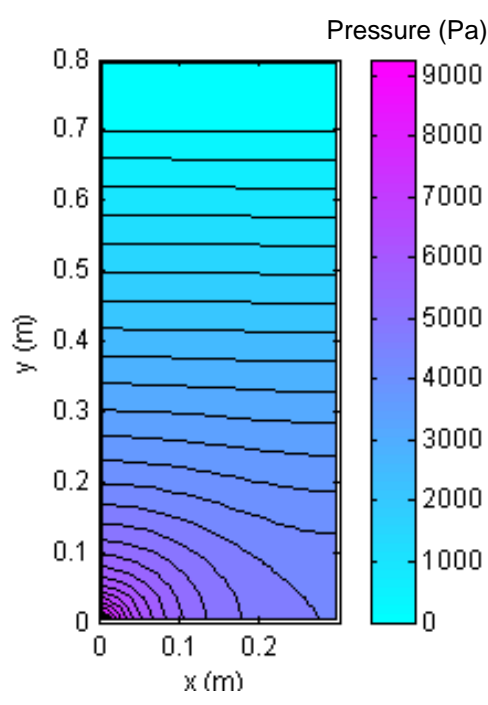

(c) time: $35 \mathrm{~s}$

Figure 3. The pressure distribution of grout at different times

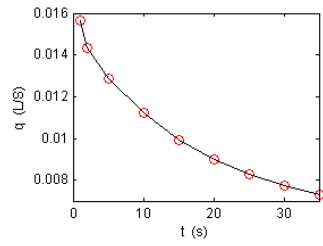

Figure 4. The variation of grout flow rate over time

\section{Conclusions}

In order to increase the understanding of the grout's two-dimensional flow behaviour in rock joints, a numerical model is developed which uses finite volume method for discretizing the governing equations of flow and volume of fluid technique for tracking the interface between grout and air. Using the model, the flow process of a Newtonian grout in a rectangular plane fissure initially filled with air is numerically investigated under a constant grouting pressure. The results show that before the flow front reached the side wall, the grout flow exhibits a centrosymmetric radial flow and the shape of the region filled by grout is approximately a circle. After that, the grout flow direction changes and on the whole it flows along the y-direction, parallel to the side wall, with an uneven flow front. With the gradual increase of grout propagation length, the flow front tends to be flat and finally almost becomes a horizontal straight line. The pressure gradient of the flow field continuously decreased with the enlargement of grout spread area, as results in a rapid decline of grout rate.

\section{Acknowledgements}

This work was financially supported by the National Natural Science Foundation of China (U1404525 and 51378473), the Natural Science Foundation of Henan Province (142300410234 and 142300413205), and Program for Innovative Research Team (in Science and Technology) in University of Henan Province (14IRTSTHN027).

\section{References}

[1] B. Amadei, W.Z. Savage, "An analytical solution for transient flow of Bingham viscoplastic materials in rock fractures", International Journal of Rock Mechanics and Mining Sciences, 2001, vol. 38, pp. 285-296.

[2] H. Stille, G. Gustafson, L. Hassler, "Application of new theories and technology for grouting of dams and foundations on rock", Geotechnical and Geological Engineering, 2012, vol. 30, pp.603-624.

[3] L. Hassler, U. Hakansson, H. Stille, "Computer-simulated flow of grouts injointed rock", Tunnelling and Underground Space Technology, 1992, vol. 7, pp. 441-446.

[4] M. Eriksson, H. Stille, J. Andersson, "Numerical calculations for prediction of grout spread with account for filtration and varying aperture", Tunnelling and Underground Space Technology, 2000, vol. 15 , pp. 353-364.

[5] M.J. Yang, Z.Q. Yue, P.K.K. Lee, B. Su, L.G. Tham, "Prediction of grout penetration in fractured rocks by numerical simulation", Canadian Geotechnical Journal, 2002, 39, pp. 1384-1394.

[6] O. Saeidi, H. Stille, S.R. Torabi, "Numerical and analytical analyses of the effects of different joint and", Tunnelling and Underground Space Technology, 2013, vol. 38, pp. 11-25.

[7] J. Funehag, G. Gustafson, "Design of grouting with silica sol in hard rock - New methods for calculation of penetration legth, Part I", Tunnelling and Underground Space Technology, 2008, vol. 23, pp. 1-8.

[8] M. El-Tani, "Grouting rock fractures with cement grout", Rock Mechanics and Rock Engineering, 2012, vol. 45, pp. 547-561.

[9] H. K. Versteeg, W. Malalasekera, "An introduction to computational fluid dynamics: The Finite Volume Method", England: Pearson Education Limited, Second edition, 2007.

[10] M. Rudman, "Volume-tracking method for interfacial flow calculation", International Journal for Numerical Methods in Fluids, 1997, vol. 24, pp. 671-691. 